\title{
Activities of Naturally Occurring Alkaloids Bulleyaconitine A
}

\author{
Shiyang Zhai, ${ }^{\dagger, a}$ Wenjing Zhou, ${ }^{\dagger, a}$ Yifan $\mathrm{Xu},{ }^{a}$ Xiaosu Wang, ${ }^{a}$ Xiao Ye, ${ }^{a}$ Shuyue Wang, ${ }^{a}$ \\ Wenhui Wu, ${ }^{a}$ and Ruihua Guo*,a,b,c,d,e \\ ${ }^{a}$ College of Food Science and Technology, Shanghai Ocean University, Shanghai 201306, China \\ ${ }^{b}$ Shanghai Engineering Research Center of Aquatic-Product Processing \& Preservation, Shanghai 201306, China \\ ${ }^{c}$ National Experimental Teaching Demonstration Center for Food Science and Engineering (Shanghai Ocean University), \\ Shanghai 201306, China \\ ${ }^{d}$ Laboratory of Quality and Safety Risk Assessment for Aquatic Product on Storage and Preservation (Shanghai), \\ Ministry of Agriculture, Shanghai 201306, China \\ ${ }^{e}$ Quality Supervision, Inspection and Testing Center for Cold Storage and Refrigeration Equipment, \\ Ministry of Agriculture, Shanghai 201306, China
}

\begin{abstract}
Bulleyaconitine A, a C19-diterpenoid alkaloid extracted from Aconitum bulleyanum Diels, showed various bioactivities, including anti-inflammatory, antinociceptivity, immunomodulatory effects as well as some toxicities. This paper reviewed the bioactivities of Bulleyaconitine A and the relevant mechanisms, toxicities, as well as the metabolism.
\end{abstract}

Keywords bulleyaconitine A, antinociceptivity, anti-inflammatory, immunomodulatory effects, toxicities

\section{Introduction}

Bulleyaconitine A with C19-diterpenoid alkaloid exhibited various bioactivities, ${ }^{[1]}$ including anti-inflammatory, analgesic activity, immunomodulatory effects, and toxicities (Figures 1). ${ }^{[2-4]}$ In 1980, bulleyaconitine A was firstly isolated from a folk herbal medicine Aconitum bulleyanum Diels, which was used for the treatment of chronic pain and rheumatoid arthritis in west Yunnan province by Kunming Institute of Botany, Chinese Academy of Science. ${ }^{[5]}$ In 1980s, bulleyaconitine A has been approved by the China Food and Drug Administration as intramuscular injections, tablets and soft gel capsules for treating chronic pain, and rheumatoid arthritis in China. ${ }^{[6]}$ To date, its dosage forms have extended to oral liquid, patches, liposomes, micro-emulsion, dripping pills, etc. ${ }^{[7-10]}$ Oral liquid is now commercially available in China while others are still in the preclinical stage. Meanwhile, its clinical applications in China have been extended to osteoarthritis, muscular fibrositis, neck and shoulder pain, low back pain, cancer pain, etc. ${ }^{[1]}$ Although its antinociceptive mechanism remained unknown at the time when it was approved by CFDA, it was discovered by King's lab in 2016. ${ }^{[11]}$ Furthermore, the metabolites and metabolic pathways of bulleyaconitine A in rat liver microsomes were investigated by Bi's group in 2014. They found 12 metabolites of bulleyaconitine A transformed by CPY450 in vitro and gained some more detailed information. As an analgesic, whose analgesic ability performs 65 and 7200 times as morphine and

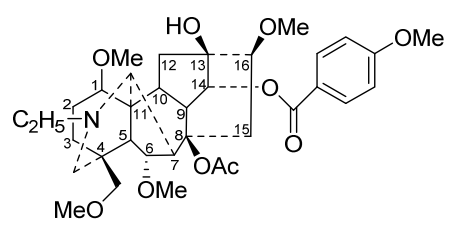

Figure 1 The structure of bulleyaconitine A. aspirin, respectively, bulleyaconitine A not only shows no tolerance but also can eliminate morphine tolerance. ${ }^{[12]}$ This review mainly focuses on the bioactivities and relevant mechanisms, toxicities, metabolisms of bulleyaconitine A, as well as some novel researches on it.

\section{Bioactivities and relevant mechanisms of bulleyaconitine $\mathrm{A}$}

\section{Antinociceptive activity}

For centuries, the Aconitum genus has been used for analgesic, antirheumatic and neurological indications in China and other Asian countries, in which bulleyaconitine A accompanied with other alkaloids, such as mesaconitine, 3-acetylaconitine, and lappaconitine, play an important role. It was initially claimed that the antinociceptive activity of bulleyaconitine A might have some relationship with the voltage-gated sodium channel because it can reduce neuronal $\mathrm{Na}^{+}$ current in a use-dependent manner and display long-acting local anesthetic properties in rats. ${ }^{[3]}$ However, according to the result of King's lab recently, ${ }^{[11]}$ bulleyaconitine A actually performs its antinociceptive activity by stimulating the dynorphin A expression in spinal microglia and performs its anti-hypersensitivity ${ }^{[13]}$ in the same way. Dynorphin is known as an analgesic biomaterial produced by the precursor protein prodynorphin expressed in spinal microglia, and it has been proved to have a strong antinociceptive activity. Surprisingly, bulleyaconitine A dose contribute to the expression of prodynorphin in spinal microglia by activating the cAMP-PKA-p38 $\beta$ CREB signaling pathway. ${ }^{[11]}$ In this pathway, the Gs-protein, cAMP/ PKA, p38 $\beta$ MAPK as well as CREB, are successively activated in the spinal microglia one by one through a series of phosphorylation, which mediates prodynorphin expression, and finally performs its antinociceptive activity and anti-hypersensitivity.

\footnotetext{
* E-mail: rhguo@shou.edu.cn; Tel.: 0086-021-61900387 Received September 29, 2017; accepted October 18, 2017. ${ }^{\dagger}$ These authors contributed equally to this work.
} 


\section{Anti-inflammatory activity}

In 1983, Tang firstly reported bulleyaconitine A showed antiinflammatory activity. ${ }^{[5]}$ In 1996, the result of a clinical study on 134 patients, in which 53 and 30 patients struggled with rheumatic arthritis and rheumatoid arthritis, respectively, showed that the application of bulleyaconitine A could relieve or even eliminate their clinical symptoms and its total effectiveness comes up to $93.3 \%{ }^{[14]}$ Besides, the combination therapies of bulleyaconitine A applied in many cases presented better anti-inflammatory or antinociceptive effects than the single application of bulleyaconitine A. For example, bulleyaconitine A combing with chondroitin sulfate was applied in the clinical treatment of periarthritis of shoulder in $1995 .{ }^{[15]}$ In 2005, bulleyaconitine A combing with high dose Danshen (salvia miltiorrhiza) was treated for clinical cancer pain. ${ }^{[16]}$ Bulleyaconitine A combing with intra-articular injection of sodium hyaluronate or Shuangbai powder or tripterygium glycosides tables was cured knee osteoarthritis in 2013 and 2015, respectively. ${ }^{[17-19]}$ In 2014, bulleyaconitine A combing with methotrexate was used for the treatment of rheumatoid arthritis.

However, the mechanism of its anti-inflammatory is unclear. In 2013, the project of "Effect of Bulleyaconitine A on Adjuvant Arthritis Rats" was conducted by Long Li's group for investigating the therapeutic effect of bulleyaconitine A on adjuvant arthritis and exploring the treatment mechanism. The result ${ }^{[20]}$ showed that 100 $\mu \mathrm{g} / \mathrm{kg}$ of bulleyaconitine A can reduce significantly the circumference of arthritic ankle in adjuvant arthritis Wistar rats. Moreover, the level of TNF- $\alpha$ and PGE2 in serum decreased in $100 \mu \mathrm{g} / \mathrm{kg}$ bulleyaconitine A treated rats $(p<0.01)$, and the level of TNF- $\alpha$ in serum also decreased in $50 \mu \mathrm{g} / \mathrm{kg}$ bulleyaconitine A group, which suggested that bulleyaconitine A can improve the inflammation of adjuvant arthritis Wistar rats by reducing the level of TNF- $\alpha$ and PGE in serum.

\section{Immunomodulatory effects}

Bulleyaconitine A used in rheumatoid arthritis (RA), possesses not only antinociceptivity and anti-inflammation, but also immunomodulatory effects. ${ }^{[1,12]} \mathrm{RA}$ is known as a long-term autoimmune disorder which involves the body's immune system attacking the joints. Bulleyaconitine A can relieve rheumatoid arthritis through its immunomodulatory effects according to Ye's report. ${ }^{[21]}$ Considering that both the specific and non-specific immune responses are involved in the formation of arthritis, bulleyaconitine A was used as a whole administration for observing its effect on some immune functions in $\mathrm{Balb} / \mathrm{c}$ mice. Balb/c mice were divided randomly into three groups including physiological saline group as a control group, bulleyaconitine A groups $(0.08,0.16,0.32 \mathrm{mg} / \mathrm{kg}$, intramuscularly) as test groups, and hydrocortisone $(10 \mathrm{mg} / \mathrm{kg})$ group as a positive control group. Mice were sacrificed after administration for $7 \mathrm{~d}$, and relevant evaluation indexes, such as spleen-index, thymus-index, lymphocytes proliferation, level of total IgG in serum, phagocytosis of peritoneal macrophages $(\mathrm{M} \varphi)$, NO in supernatants of macrophages, were tested and recorded by different methods. Bulleyaconitine A $(0.32 \mathrm{mg} / \mathrm{kg})$ inhibited T- and B-lymphocytes proliferations and reduced NO in supernatants, bulleyaconitine A $(0.16,0.32 \mathrm{mg} / \mathrm{kg})$ decreased the thymus-index and the level of total IgG in serum, and bulleyaconitine $\mathrm{A}(0.08,0.16,0.32 \mathrm{mg} / \mathrm{kg})$ inhibited the phagocytosis of peritoneal macrophages $(\mathrm{M} \varphi)$. The result indicates that bulleyaconitine A has a suppressive effect on some immune functions of $\mathrm{Balb} / \mathrm{c}$ mice.

\section{Toxicities of bulleyaconitine A}

Bulleyaconitine A also has some toxicities despite its strong antinociceptivity and approval of China Food and Drug Administration. The toxicities of bulleyaconitine A have always been concerned since $1980 \mathrm{~s}$, and many research on it has been done to show its clinical safety. In 1986, a toxicologic research on mice and rats was conducted by Tang's group. Both mice and rats were injected poisoning-dose bulleyaconitine $\mathrm{A}$, whose values are 10-40 times as the clinical application dose. After injection, toxic symptoms appeared, including salivation, vomiting, continuing convulsions, respiratory depression, and even the development of asphyxia death. Dead ones were within $12 \mathrm{~h}$ after administration and survivors were recovered after $24 \mathrm{~h} .{ }^{[5]}$ Wang et al. found that bulleyaconitine A can induce acute systemic toxicity in rats, including hyperexcitability, arrhythmia, sedation, and respiratory distress. They also made some attempts to reduce this kind of systemic toxicity by co-administration of lidocaine or epinephrine and it did work. Finally, it was concluded that the systemic toxicity of bulleyaconitine A could be minimized by reducing blood flow at the injected site. ${ }^{[3]}$ There are also many side effects in clinical research, such as dizziness, nausea, general sensation, palpitations, numbness, body itching and bilateral lower extremity urticaria, sweating, dry mouth, injection site swelling, allergic reaction, distinct feeling of pain at injection site, etc. But they were tolerable and would disappear after some time. ${ }^{[4,5,22]}$ It is possible that the biological activity of bulleyaconitine A may be caused by its toxicity rather than a specific pharmacological action. ${ }^{[23]}$ However, King's group found that the anti-hypersensitivity of bulleyaconitine A can be separated from its neurotoxicity by the mechanism of action, ${ }^{[24]}$ and the relatively wide therapeutic window of bulleyaconitine A suggests that its antinociception could be separated from its toxicity. ${ }^{[13]}$ It means that bulleyaconitine A performs its ability by a specific pharmacological action in some way rather than its toxicity.

\section{Metabolites and metabolic pathways of bulleyaconitine $A$}

The metabolites of drugs are always being concerned, and they cause the side effects or perform its toxicities. The research on the metabolites and the metabolic pathways of bulleyaconitine A plays an important role during the period of explaining the bioactivities and toxicities of bulleyaconitine A. In 2013, the metabolites and the metabolic pathways of bulleyaconitine $\mathrm{A}$ in rat liver microsomes were investigated by Bi's group, twelve metabolites of bulleyaconitine A were identified and some important CYP isoforms contributing to the metabolism of bulleyaconitine A were discovered. Bulleyaconitine A was added into the prepared microsomal suspension containing NADPH and specific CYP inhibitors $(\alpha$-naphthoflavone for CYP1A2, quinidine for CYP2D, diethyldithiocarbamate for CYP2E, ketoconazole for CYP3A and sulfaphenazole for CYP2C), then acetonitrile was added after the incubation of the suspension to stop the reaction. ${ }^{[6]}$ Finally, the supernatant was analyzed using HPLC-MS after centrifuging for some time. The results are shown in Figure 2 and Table 1.

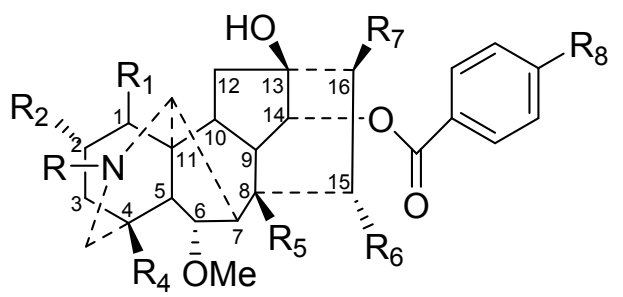

Figure 2 The structures metabolites of bulleyaconitine A.

The results indicated that bulleyaconitine A is mainly metabolized by $\mathrm{CPY} 3 \mathrm{~A}$ and $\mathrm{CPY} 2 \mathrm{C}$ in rat microsomes. Moreover, reactions happened on its side chains, including demethylation, de-ethylation, hydroxylation, didemethylation, deacetylation and dehydration. 
Table 1 The structures metabolites of bulleyaconitine A

\begin{tabular}{cccccccccc}
\hline Metabolites & $\mathrm{R}$ & $\mathrm{R}_{1}$ & $\mathrm{R}_{2}$ & $\mathrm{R}_{4}$ & $\mathrm{R}_{5}$ & $\mathrm{R}_{6}$ & $\mathrm{R}_{7}$ & $\mathrm{R}_{8}$ & Enzymes \\
\hline $\mathrm{M} 1$ & $-\mathrm{C}_{2} \mathrm{H}_{5}$ & $-\mathrm{OMe}$ & $-\mathrm{H}$ & $-\mathrm{C}_{2} \mathrm{H}_{5} \mathrm{O}$ & $-\mathrm{OAc}$ & $-\mathrm{H}$ & $-\mathrm{OH}$ & $-\mathrm{OMe}$ & CYP3A, 2C, 2D, 2E1 \\
$\mathrm{M} 2$ & $-\mathrm{C}_{2} \mathrm{H}_{5}$ & $-\mathrm{OH}$ & $-\mathrm{H}$ & $-\mathrm{C}_{2} \mathrm{H}_{5} \mathrm{O}$ & $-\mathrm{OAc}$ & $-\mathrm{H}$ & $-\mathrm{OH}$ & $-\mathrm{OMe}$ & Transformed by M1 or M3 \\
$\mathrm{M} 3$ & $-\mathrm{C}_{2} \mathrm{H}_{5}$ & $-\mathrm{OH}$ & $-\mathrm{H}$ & $-\mathrm{C}_{2} \mathrm{H}_{5} \mathrm{O}$ & $-\mathrm{OAc}$ & $-\mathrm{H}$ & $-\mathrm{OMe}$ & $-\mathrm{OMe}$ & CYP3A, 2C, 2E1 \\
M4 & $-\mathrm{C}_{2} \mathrm{H}_{5}$ & $-\mathrm{OMe}$ & $-\mathrm{H}$ & $-\mathrm{C}_{2} \mathrm{H}_{5} \mathrm{O}$ & $-\mathrm{OH}$ & $-\mathrm{H}$ & $-\mathrm{OMe}$ & $-\mathrm{OMe}$ & CYP3A, 2C, 2D, 2E1 \\
M5 & $-\mathrm{C}_{2} \mathrm{H}_{5}$ & $-\mathrm{OMe}$ & $-\mathrm{H}$ & $-\mathrm{H}$ & $-\mathrm{OAc}$ & $-\mathrm{H}$ & $-\mathrm{OMe}$ & $-\mathrm{OMe}$ & CYP3A, 2C \\
M6 & $-\mathrm{C}_{2} \mathrm{H}_{5}$ & $-\mathrm{OMe}$ & $\Delta^{2,3}$ & $-\mathrm{C}_{2} \mathrm{H}_{5} \mathrm{O}$ & $-\mathrm{OAc}$ & $-\mathrm{H}$ & $-\mathrm{OMe}$ & $-\mathrm{OMe}$ & CYP3A, 2C \\
M7 & $-\mathrm{C}_{2} \mathrm{H}_{5}$ & $-\mathrm{OMe}$ & $-\mathrm{OH}$ & $-\mathrm{C}_{2} \mathrm{H}_{5} \mathrm{O}$ & $-\mathrm{OAc}$ & $-\mathrm{H}$ & $-\mathrm{OMe}$ & $-\mathrm{OMe}$ & CYP3A, 2C, 2D \\
M8 & $-\mathrm{C}_{2} \mathrm{H}_{5}$ & $-\mathrm{OMe}$ & $-\mathrm{H}$ & $-\mathrm{C}_{2} \mathrm{H}_{5} \mathrm{O}$ & $-\mathrm{OAc}$ & $\Delta^{15,16}$ & $\Delta^{15,16}$ & $-\mathrm{OMe}$ & CYP3A, 2C \\
M9 & $-\mathrm{C}_{2} \mathrm{H}_{5}$ & $-\mathrm{OMe}$ & $-\mathrm{H}$ & $-\mathrm{C}_{2} \mathrm{H}_{5} \mathrm{O}$ & $-\mathrm{OAc}$ & $-\mathrm{OH}$ & $-\mathrm{OMe}$ & $-\mathrm{OMe}$ & CYP3A, 2C, 2D \\
M10 & $-\mathrm{C}_{2} \mathrm{H}_{5}$ & $-\mathrm{OMe}$ & $-\mathrm{H}$ & $-\mathrm{C}_{2} \mathrm{H}_{5} \mathrm{O}$ & $-\mathrm{OAc}$ & $-\mathrm{H}$ & $-\mathrm{OMe}$ & - & CYP3A,2C \\
M11 & $-\mathrm{C} 2_{2} \mathrm{H}_{5}$ & $-\mathrm{OMe}$ & $-\mathrm{H}$ & $-\mathrm{C}_{2} \mathrm{H}_{5} \mathrm{O}$ & $-\mathrm{OAc}$ & $-\mathrm{H}$ & $-\mathrm{OMe}$ & $-\mathrm{OMe}$ & CYP3A, 2C, 1A2, 2E1 \\
M12 & - & $-\mathrm{OMe}$ & $-\mathrm{H}$ & $-\mathrm{C}_{2} \mathrm{H}_{5} \mathrm{O}$ & $\Delta^{8,15}$ & $\Delta^{8,15}$ & $-\mathrm{OMe}$ & $-\mathrm{OMe}$ & CYP3A, 2C, 2D, 2E1 \\
\hline
\end{tabular}

\section{Determination and quantification of bulley- aconitine $\mathrm{A}$ in vivo}

Liquid chromatography and mass spectrometry play an important role in the determination and quantification of bulleyaconitine A in vivo. The extreme low concentration of bulleyaconitine A for its low dose and rapid metabolism results in great difficulties for developing the analytical methods. Before 2004, none of reported methods for the determination of bulleyaconitine $\mathrm{A}$ in blood or urine is validated or sensitive enough for the pharmacokinetic study in humans, including high-performance liquid chromatography with UV detection (HPLC-UV), high-performance liquid chromatography with mass spectrometry detection (HPLC-MS) and gas chromatography with mass spectrometry detection (GC-MS) ${ }^{[25]}$ In 2004, HPLC-MSMS was established to determine bulleyaconitine $\mathrm{A}$ in human plasma by Weng's group. Their clinical research presented that this method was rapid and practically applicable to the pharmacokinetic studies of bulleyaconitine $\mathrm{A}$ in human. ${ }^{[25]}$ In 2009, an in-depth research was performed using this method by Xiong's group. Using a simple protein precipitation procedure for the quantification of bulleyaconitine A in human serum, they developed a sensitive, specific, simple, and rapid LC-MS-MS method, which allows the assay of up to 100 samples per day, and avoids using a large volume of plasma and a laborious liquid-liquid extraction procedure in Weng's research. With this method, only $0.2 \mathrm{~mL}$ serum is needed for analysis and it allows quantification of bulleyaconitine A ranging from 0.0587 to $11.7 \mathrm{ng} / \mathrm{mL} .^{[26]}$ The quantification of bulleyaconitine A of 0.0587 $\mathrm{ng} / \mathrm{mL}$ is reported to be sensitive enough to use for the pharmacokinetic study of intramuscularly injected bulleyaconitine A. However, this value was not sensitive enough for a pharmacokinetic study of orally administered bulleyaconitine A in human plasma. Therefore, a more sensitive LC-MS-MS method was developed for the quantification of bulleyaconitine A in rat plasma and it was applied to an oral pharmacokinetic study of bulleyaconitine A in rats by Wang's group in 2012. The oral pharmacokinetic data for bulleyaconitine A were firstly reported, suggesting that this method was valuable for future clinical studies. ${ }^{[27]}$

\section{Conclusions}

The research on bulleyaconitine $\mathrm{A}$ is involved in bioactivities, mechanisms, toxicities, metabolisms, dose forms, combination therapy, determination and quantification as well as clinical research. Bulleyaconitine A has strong antinociceptive activity valued 65 times as morphine without tolerance, and the mechanism of its antinociceptive activity is clarified. However, the mechanism of its toxicities still remains unknown since its antinociceptive activity could be separated from its toxicities. Therefore, more research on the mechanism of its toxicities should be performed. Besides, more research on the pharmaceutics and medicinal chemistry of bulleyaconitine A to reduce its toxicities seems to be valuable. As an excellent but not that perfect analgesic, bulleyaconitine A needs more concern.

\section{Acknowledgement}

The work was supported by the National Natural Science Foundation of China (No. 81502955), the Doctoral Scientific Research Foundation of Shanghai Ocean University (No. A2-030214-300077), and the Young Teachers Training Program of Shanghai (No. A1-2035-15-0021-101), the Shanghai High Technology Research and Development Program (Nos. 14431906000 and 15410722500).

\section{References}

[1] Yang, X. L.; Yang, D. N.; Yang, Q. R.; Zhu, K.; Zhang, Y. S. World Clin. Drugs 2013, 34, 80.

[2] Wang, C. F.; Gerner, P.; Schmidt, B.; Xu, Z. Z.; Nau, C.; Wang, S. Y.; Ji, R. R.; Wang, G. K. Anesth. Analg. 2008, 107, 1397.

[3] Wang, C. F.; Gerner, P.; Wang, S. Y.; Wang, G. K. Anesthesiology 2007, $107,82$.

[4] Weng, W. Y.; Xu, H. N.; Huang, J. M.; Wang, G. Q.; Shen, T.; Zhang, J. F. Biol. Pharm. Bull. 2005, 28, 747.

[5] Tang, X, CH. Chin. J. New Drugs Clin. Rem. 1986, 5, 120.

[6] Bi, Y. F.; Zhuang, X. Y.; Zhu, H. B.; Song, F. R; Liu, Z. Q.; Liu, S. Y. Biomed. Chromatogr. 2015, 29, 1027.

[7] Weng, W. Y. Master's Thesis, Transdermal Characteristic and Transdermal Delivery System of Bulleyaconitine A, Fudan University, Shanghai, 2003, p. 3.

[8] Xiao, R. L. Master's Thesis, The Study of Drug Delivery for Bulleyaconitine A-Multivesicular Liposome and Bulleyaconitine A-DSPE-PEG2000 Micelle, Shenyang Pharmaceutical University, Shenyang, 2008, p. 4.

[9] Wu, X. H. Master's Thesis, The Study on Microemulsion of Bulleyaconitine A and Its Waterborne Substrate Transdermal Drug Delivery System, Chinese Academy of Medical Science \& Peking Union Medical College, Beijing, 2006, p. 8.

[10] Duan, S. F.; Shi, J. F. J. Pharm. Pract. 2013, 31, 217.

[11] Li, T. F.; Wu, H. Y.; Wang, Y. R.; Li, X. Y.; Wang, Y. X. Sci. Rep. 2017, 7, 1

[12] Zhang, X.; Zhou, B.; Lu, H. L. Chron. Pathematol. J. 2016, 17, 1210.

[13] Li, T. F.; Fan, H.; Wang, Y. X. J. Pain 2016, 17, 530.

[14] Chen, X, Y.; Xia, X. L.; Xiong, J. M.; Tai, P. R. Yunnan J. Tradit. Chin. Med. Mater. Medica 1996, 7, 54.

[15] Wang, Z. S.; Zhang, X. P. J. Pain 1995, 3, 120.

[16] Zheng, X. H. Clin. Med. 2005, 25, 65.

[17] Pan, G. X.; Du, D. P. Shanghai Med. J. 2013, 36, 523

[18] Xiao, W.; Lin, D. D. Clin. J. Chin. Med. 2015, 7, 1.

[19] Du, N.; Zhu, D. Y. J. Henan Univ. (Med. Sci.) 2015, 34, 50.

[20] Long, L.; Zhao, J. X.; Jia, R. L.; Liu, Y. Y.; Han, L.; Li, Z. G. Clin. Medication J. 2013, 11, 16.

[21] Lu, Y.; Han, L.; Li, H.; Weng, W. Y. Chin. J. New Drug Clin. Rem. 2007, 26, 755.

[22] Yang, C. H. Med. Pharm. Yunnan 1995, 16, 75.

[23] Bello-Ramírez, A. M.; Nava-Ocampo, A. A. Fund. Clin. Pharmacol. 2004, 18, 699 .

[24] Li, T. F.; Gong, N.; Wang, Y. X. Front. Pharmacol. 2016, 7, 1.

[25] Weng, W. Y.; Xu, H. N.; Huang, J. M.; Wang, G, Q.; Shen, T.; Zhang J. F. J. Chromatogr. B 2005, 816, 315.

[26] Xiong, X.; Zhai, S. D.; Yao, Z. Q. Biosci. Biotechnol. Biochem. 2009, 73, 1527.

[27] Wang, Q.; Tan, B.; Gong, Y. J.; Ji, G. X.; Zhang, Y.; Yang, P.; Li, W.; Shen, T. J. Pharm. Biomed. Anal. 2012, 71, 202. 